\title{
Relationship between serum neopterin levels and coronary heart disease
}

\author{
Z.Y. Liu and Y.D. Li \\ Department of Cardiology, Nanyang Central Hospital, Nanyang, China \\ Corresponding author: Y.D. Li \\ E-mail: yudonglicn@yeah.net
}

Genet. Mol. Res. 12 (4): 4222-4229 (2013)

Received January 18, 2013

Accepted July 31, 2013

Published October 7, 2013

DOI http://dx.doi.org/10.4238/2013.October.7.8

\begin{abstract}
The relationship between serum neopterin levels and coronary heart disease (CHD) was investigated. Eighty-six CHD patients were divided into an acute myocardial infarction (AMI) group $(\mathrm{N}=21)$, an unstable angina pectoris (UAP) group $(\mathrm{N}=35)$, and a stable angina pectoris (SAP) group $(\mathrm{N}=30)$, based on coronary angiography (CAG), 30 subjects without CHD served as the control group. Serum neopterin levels were determined by enzyme linked immunosorbent assay (ELISA), and relationships between neopterin and the severity of stenosis, stenosis number, and the stability of coronary artery were analyzed. Serum neopterin levels were higher in the AMI and UAP groups than in the SAP and control groups $(\mathrm{P}<$ 0.01 ), but no significant differences were observed between the AMI and UAP groups or between the SAP and control groups $(\mathrm{P}>0.05)$. Mean serum neopterin levels were higher in the single, double, and three vessel lesion groups than in the control group $(\mathrm{P}<0.05)$, whereas there were no significant differences among the lesion groups $(\mathrm{P}>$ $0.05)$. Serum levels of neopterin were significantly higher in the type II than in the type I or type III, plaque groups $(\mathrm{P}<0.01)$, the incidence of type II plaque was significantly higher in the AMI and UAP groups compared to the SAP group $(\mathrm{P}<0.01)$. Neopterin likely plays a role in the occurrence and development of athermanous plaque and can serve
\end{abstract}


as a useful biomarker of vulnerable plaques. Immunoreaction may be involved in the pathophysiological process of CHD.

Key words: Neopterin; Coronary artery; Coronary heart disease

\section{INTRODUCTION}

Coronary heart disease (CHD) is the most common type of organ lesions caused by atherosclerosis, and is a cardiovascular disease with serious hazards to human health. Acute coronary syndrome (ACS) is the serious clinical manifestation of CHD. The main mechanism of ACS is based on coronary atherosclerotic lesions, in which vulnerable plaques appear, crack, erode, rupture, and result in secondary platelet aggregation and thrombosis, which causes either complete or incomplete coronary artery occlusion (Depré et al., 1997).

ACS pathogenesis is currently a popular research topic. The occurrence of ACS is closely related to plaque vulnerability and is disproportionate to the degree of coronary artery stenosis (Naghavi et al., 2003). Coronary angiography data show that 60 to $70 \%$ of ACS had occurred in patients with mild to moderate coronary atherosclerotic lesions; however, patients with similar coronary artery stenosis severity have significantly different clinical presentations and prognoses. Some patients with severe vascular disease or complete occlusion showed only mild clinical symptoms without myocardial infarction. This suggests that the clinical onset of CHD is not dependent on the degree of stenosis, but instead depends on whether the structure of the plaque is stable. Once patients with CHD suffer from ACS, damage is severe and mortality rates are high. Therefore, the identification of vulnerable plaque, blood, and myocardium, as well as the identification of vulnerable patients, should be research priorities for cardiovascular disease (Naghavi et al., 2003). In addition, determining chemical markers related to vulnerable plaque, the timely identification of vulnerable plaques, and early prediction of ACS are of great significance to reduce the incidence of ACS and associated mortality rates.

Recent studies have suggested that immune system activation plays a role in the pathogenesis of ACS (Curtiss et al., 2000). When animals' respiratory tracts are infected with Chlamydia pneumonia, pathological changes may occur in their cardiovascular tissues with the participation of the immune system (Fan et al., 1999). Furthermore, phenotypes of lymphocytes and monocytes change in patients with atherosclerosis, as B1 and B2 integrant, which bind readily to inflammatory cells, are expressed on the cell surface. Transgenic animal experiments have demonstrated confirm that $\mathrm{T}$ lymphocytes and $\mathrm{B}$ lymphocytes play a pivotal role during the atherosclerotic process (Schmitz et al., 1998). The number of macrophages and activated lymphocytes increases significantly in vulnerable plaque, which can make atherosclerotic plaques even more vulnerable. Once the macrophages are activated by $\gamma$-interferon, they can synthesize neopterin. Therefore, neopterin can act as a sensitive marker of cell-mediated immune activation (Huber et al., 1984). The concentration of neopterin increases in patients with diseases related to cell-mediated immune activation (Wirleitner et al., 2002) and significantly increases in circulating blood of acute inflammation patients (Avanzas et al., 2004). The presents study aimed to investigate the relationship between neopterin and CHD. Neopterin levels were compared between in patients with CHD that varied in the stenosis degree of coronary artery disease, the number of diseased vessels, and plaque stability. 


\section{MATERIAL AND METHODS}

\section{Research subjects}

Eighty-six patients with CHD, including 49 men and 37 women, with an average age of $59.3 \pm 10.2$ years, were recruited from the Department of Cardiology, Nanyang Central Hospital. All CHD diagnoses were confirmed by coronary angiography based on the luminal diameter stenosis of no less than $50 \%$ in at least. One of the left main, left anterior descending artery, left circumflex artery, or right coronary artery (Gibbons et al, 1999). The 86 patients with CHD were divided into three groups: 21 patients were in the acute myocardial infarction (AMI) group, 35 patients were in the unstable angina pectoris (UAP) group, and 30 patients were in the stable angina pectoris (SAP) group. A further, 30 subjects, whose average age was $57.3 \pm 11.2$ years, with a normal CAG result were chosen as the control group, including 17 men and 13 women. Patients with other heart diseases, such as cardiomyopathy and valvular disease, as well as tumors, infections, systemic autoimmune diseases, liver and renal dysfunction, and significant cerebrovascular or peripheral vascular diseases were excluded. Differences in sex, age, smoking, hypertension, family history and blood lipid levels among groups were not statistically significant $(\mathrm{P}>0.05)$.

\section{Specimen collection}

Three milliliters fasting venous blood was collected from patients in each group within $24 \mathrm{~h}$ after hospitalization, except for patients with AMI and UAP, who were sampled immediately upon hospitalization). All specimens were centrifuged at $1600 \mathrm{rpm}$ for $5 \mathrm{~min}$ within $30 \mathrm{~min}$ of collection. The serum was then separated and tubes were sealed. Specimens were stored at $-30^{\circ} \mathrm{C}$.

\section{Detection of neopterin}

Neopterin detection was carried out with enzyme linked immunosorbent assay (ELISA) using the IBL ELISA kit (Germany) in strict accordance with manufacturer instructions.

\section{Coronary angiography, number of diseased coronary arteries, and plaque morphological classification}

Using Judi kin's catheter technology, coronary angiography was accomplished through the right femoral artery or the radial artery approach by conventional multi-position projection. Angiography results were analyzed by two or more experienced physicians. The maximum luminal diameter stenosis percentage, which showed the most serious stenosis, was calculated according to the angiography image in several projection positions. The left main, left anterior descending artery, left circumflex artery, and right coronary arteries were analyzed and then patients were sub-divided into the one, two, or three diseased vessels groups. Main vessels, such as diagonal vessels and edge vessels, were classified together for statistical analyses. Based on the plaque morphological classification of Ambrose (Ambrose et al., 1985), coronary atherosclerotic lesions were divided into three types. Type I lesions show a 
smooth surface and wider basement, and are centripetal or eccentric lesions. Type II lesions have narrow basements or a closed angle shape (smooth or rough surface), a niche, or a rough surface or crater shape (centripetal or eccentric). Type III lesions are agree segmented irregular stenosis lesions.

\section{Statistical analysis}

The statistical software package SPSS10.0 was used for all analyses. Measurement data is expressed as means \pm standard deviation. The Student test and the chi-squared test were used for between group comparisons. $\mathrm{P}<0.05$ represented statistically significant

\section{RESULTS}

\section{Comparison of neopterin concentrations in serum between CHD subunits and control group}

Neopterin concentrations in the CHD groups and the control group are shown in Table 1. Neopterin concentrations in the serum of patients in the AMI and UAP groups were significantly higher than those of the SAP and control group $(\mathrm{P}<0.01)$, but there was no statistical significance between the AMI and UAP groups, or between the SAP and control groups $(\mathrm{P}>0.05)$.

Table 1. Contrasts of neopterin concentration in serum between CHD subunits and control group (means $\pm \mathrm{SD}$ ).

\begin{tabular}{llr}
\hline Groups & N & Neopterin (nM) \\
\hline AMI & 21 & $11.85 \pm 2.30$ \\
UAP & 35 & $9.94 \pm 1.91^{\mathrm{a}}$ \\
SAP & 30 & $8.13 \pm 2.10^{\text {bc }}$ \\
Control & 30 & $7.97 \pm 1.99^{\text {bcd }}$ \\
\hline
\end{tabular}

$\mathrm{AMI}=$ acute myocardial infarction; $\mathrm{UAP}=$ unstable angina pectoris; $\mathrm{SAP}=$ stable angina pectoris. Compared with AMI group: ${ }^{\mathrm{a}} \mathrm{P}>0.05$; ${ }^{\mathrm{b}} \mathrm{P}<0.01$, compared with UAP group: ${ }^{\mathrm{C}} \mathrm{P}<0.01$, compared with $\mathrm{SAP}$ group: ${ }^{\mathrm{d}} \mathrm{P}>0.05$.

\section{Comparison of neopterin concentration in serum between groups varying in diseased coronary artery numbers and control group}

Neopterin concentrations in the coronary artery severity groups are shown in Table 2. Neopterin concentrations in serum of patients with CHD in the one, two, and three diseased vessels groups were significantly higher than that of the control group $(\mathrm{P}<0.05)$. There were no statistically significant differences among the three diseased vessel groups $(\mathrm{P}>0.05)$.

$\begin{aligned} & \text { Table 2. Contrasts of neopterin concentration in serum between groups with different diseased coronary artery } \\
& \text { numbers and control group (means } \pm \mathrm{SD}) .\end{aligned}$
\begin{tabular}{lcc}
\hline Groups & $\mathrm{N}$ & Neopterin $(\mathrm{nM})$ \\
\hline One diseased vessel & 43 & $9.89 \pm 1.28$ \\
Two diseased vessels & 22 & $10.07 \pm 1.81^{\mathrm{a}}$ \\
Three diseased vessels & 20 & $9.93 \pm 1.91^{\mathrm{ac}}$ \\
Control & 30 & $7.97 \pm 1.99^{\text {bde }}$ \\
\hline Compared with one diseased vessel group: ${ }^{\text {a }} \mathrm{P}>0.05 ;{ }^{\mathrm{b}} \mathrm{P}<0.05$, compared with two diseased vessels group: ${ }^{\mathrm{c}} \mathrm{P}>$ \\
$0.05,{ }^{\mathrm{d}} \mathrm{P}<0.05$, compared with three diseased vessels group: ${ }^{\mathrm{e}} \mathrm{P}<0.05$.
\end{tabular}




\section{Comparison of neopterin concentration in serum between groups with different coronary artery plaque shapes and control group}

Neopterin concentrations of different plaque types are shown in Table 3. Neopterin concentrations in serum of type I, type II, and type III patients were higher than that of the control group. Furthermore, neopterin concentrations in serum of patients in the type II group were higher than those of the type I and type III groups.

\begin{tabular}{l}
$\begin{array}{l}\text { Table 3. Contrasts of neopterin concentration in serum between groups with different coronary artery plaque } \\
\text { shapes and control group (means } \pm \text { SD). }\end{array}$ \\
\hline Groups \\
\hline Type I plaque
\end{tabular}

Compared with Type I and Type III plaque groups: ${ }^{*} \mathrm{P}<0.01$, compared with Type I plaque group: ${ }^{\#} \mathrm{P}>0.05$.

\section{Comparison of coronary artery disease plaque shapes among CHD subunits}

Results of the frequencies of plaque shapes in CHD patients are shown in Table 4. The incidence of Type II plaques was significantly higher in the AMI and UAP groups than in the SAP group, whereas type I and type III plaques were lower in the AMI and UAP groups than in the SAP group $(\mathrm{P}<0.01)$.

\begin{tabular}{l}
\multicolumn{4}{l}{ Table 4. Contrast of incidence of different coronary artery disease plaque shape among CHD subunits. } \\
\begin{tabular}{lcccc}
\hline Groups & $\mathrm{N}$ & Different plaque types (\%) & \\
\cline { 2 - 4 } & & Type I plaque & Type II plaque & Type III plaque \\
\hline AMI & 21 & $4(19.05)$ & $3(61.90)$ & $4(19.05)$ \\
AUP & 35 & $7(20.00)^{*}$ & $20(57.14)^{* \#}$ & $8(22.86)^{*}$ \\
SAP & 30 & $10(33.33)^{\Delta}$ & $5(16.67)^{\Delta}$ & $15(50.00)^{\Delta}$ \\
\hline
\end{tabular}
\end{tabular}

Compared with AMI group: $* \mathrm{P}>0.05$, compared with SAP group: ${ }^{*} \mathrm{P}<0.01$, compared with AMI group and UAP group: $\triangle \mathrm{P}<0.01$. For abbreviations, see legend to Table 1 .

\section{DISCUSSION}

The association between inflammation and atherosclerosis has received increasing attention in recent years. More and more studies have demonstrated that local and systemic inflammation plays an important role in the occurrence and development of atherosclerosis, as well as in its related complications. A variety of inflammatory cells (e.g., coenocytes, macrophages, T-lymphocytes), cytokines, adhesion molecules, and growth factors are involved in the formation and development of atherosclerosis. In addition, these cells may play an important role in the early initiation of ACS.

The main determinants of plaque stability, such as lipid core size, fibrous cap thickness, and damage repair capacity, are all related to the inflammatory response, especially the number and activity of monotypes macrophages. The major lipid components, including cholesterol and cholesterol ester, make their way into plaques, which is the result of the common 
role of inflammatory cells. Low-density lipoproteins (LDLs) and coenocytes each combine with dysfunction of endothelial cell adhesion The former are modified to oxidized low density lipoproteins (OX-LDLs), whereas the latter evolve into macrophages and express scavenger receptors, engulf lipid into foam cells, and release lipids after their death, which then constitutes the core of the plaque. Inflammatory cells can reduce the thickness of the fibrous cap in three ways (Weissberg, 2000): i) mononuclear macrophages can produce metal protease (MMPs), which can specifically combine with extracellular matrix components, which leads to fiber cap matrix degradation; ii) $\gamma$-interferon and other cytokines can inhibit proliferation and collagen synthesis of vascular smooth muscle cells (VSMC), and can further promote the expression of MMP genes; iii) activated macrophages can induce VSMC apoptosis.

Several mononuclear macrophages and T lymphocytes have been shown to infiltrate and they are activated in unstable plaque and ruptured plaque. The extent of such infiltration is significantly higher than in stable plaque, and is positively correlated with disease severity and patient prognoses (Ueland et al., 2006). Urgency of clinical onset of CHD is not dependent on the stenosis degree, but instead depends on the structural stability of plaque. Once patients with CHD suffer from ACS, there will be great harm and high mortality. Therefore, determining chemical markers related to vulnerable plaque, timely identification of vulnerable plaques, and early prediction of ACS is of great significance to reduce the incidence of ACS and associated mortality rates. To date, several markers, such as C-reactive protein (CRP) and interleukin (IL), have been applied in clinical experiments. However, these markers are all systemic and are non-specific.

Neopterin is the most important pteridine in body fluids. It is an intermediate metabolite in guanosine triphosphate metabolism and in tetrahydrobiopterin biosynthesis and it can be used as a hydroxylation cofactor of several neurotransmitter enzymes, such as phenylalanine and tyrosinase. Neopterin is produced by mononuclear macrophages, which are excited by $\gamma$-interferon that is secreted by activated T lymphocytes. Therefore, neopterin levels directly reflect the activation of macrophages, $\mathrm{T}$ lymphocytes, and cellular immunity (Weissberg, 2000). Since the concentration of neopterin reflects the level of $\gamma$-interferon secreted by activated $\mathrm{T}$ lymphocytes, which in turn directly reflects the condition of cellular immunity function in the body (Caligiuri et al., 1998), the concentration of neopterin can be detected to assess cellular immunity function. Due to its high chemical stability and low molecular weight, neopterin can easily penetrate into the blood circulation. Furthermore, the conditions of neopterin do not change in damaged areas, as do other substances with active chemical properties and high molecular weights. The neopterin level of peripheral blood can be detected to reflect the neopterin concentration of coronary artery blood, which can be used to assess the character and degree of coronary artery lesions.

Results of the present study revealed that neopterin concentrations of the AMI and UAP groups were significantly higher than those of the SAP and control groups, whereas the difference between the AMI and UAP groups was not statistically significant, nor was the difference between the SAP and control groups. These results suggest that activation of the mononuclear phagocyte system plays an important role in the onset of CHD. Plaque rupture induced by inflammatory mediators often occurs in patients with AMI. Ruptured plaques can further adsorb activated mononuclear macrophages and other inflammatory cells, which leads to a massive release of neopterin and other inflammatory markers. Neopterin activates endogenous and inducible nitric oxide syntheses to regulate the intracellular redox state (Glynn et 
al., 2000; Domanski et al., 2001) and to promote the transcription of the NF- $\kappa B$ subunit in the nucleus (Werle et al., 2002). The cell-mediated immune response is one type of atherosclerosis pathogenesis.

This study also demonstrated that the difference in neopterin concentration among the one, two, and three diseased vessels groups was not statistically significant, which suggests that neopterin is not related to the number of diseased coronary arteries, and that the coronary angiography shows the shape of plaque rather than its internal structure and properties. Due to its own limitation, coronary angiography can not directly reflect the property of plaque. Therefore, vulnerability should not be determined solely on the basis of the stenosis degree and the extent revealed by coronary angiography, but should instead be based on the recognized "gold standard, which should be complemented via intracoronary ultrasound as well as the detection of serum markers and other substances.

In the present study, we found that type II plaque was often seen in patients with AMI or UAP, while type I and type III plaques were often seen in SAP patients. This result is similar to those of Monroe et al. (2002). Plaque morphological features may indirectly reflect the stability of the plaque. Type II plaque often represents morphological changes in ACS plaque, while type I and type III plaque reflect changes in SAP plaque. This study demonstrated that the neopterin concentration of the type II plaque group was significantly higher than those of type I and type III plaque groups, which suggests that neopterin is related to unstable plaque. Therefore, neopterin can be efficiently used as a marker for the assessment of plaque stability.

\section{REFERENCES}

Ambrose JA, Winters SL, Stern A, Eng A, et al. (1985). Angiographic morphology and the pathogenesis of unstable angina pectoris. J. Am. Coll. Cardiol. 5: 609-616.

Avanzas P, Arroyo-Espliguero R, Cosin-Sales J, Quiles J, et al. (2004). Prognostic value of neopterin levels in treated patients with hypertension and chest pain but without obstructive coronary artery disease. Am. J. Cardiol. 93: 627-629.

Caligiuri G, Liuzzo G, Biasucci LM and Maseri A (1998). Immune system activation follows inflammation in unstable angina: pathogenetic implications. J. Am. Coll. Cardiol. 32: 1295-1304.

Curtiss LK, Kubo N, Schiller NK and Boisvert WA (2000). Participation of innate and acquired immunity in atherosclerosis. Immunol. Res. 21: 167-176.

Depré C, Wijns W, Robert AM, Renkin JP, et al. (1997). Pathology of unstable plaque: correlation with the clinical severity of acute coronary syndromes. J. Am. Coll. Cardiol. 30: 694-702.

Domanski M, Norman J, Wolz M, Mitchell G, et al. (2001). Cardiovascular risk assessment using pulse pressure in the first national health and nutrition examination survey (NHANES I). Hypertension 38: 793-797.

Fan Y, Wang S and Yang X (1999). Chlamydia trachomatis (mouse pneumonitis strain) induces cardiovascular pathology following respiratory tract infection. Infect. Immun. 67: 6145-6151.

Gibbons RJ, Chatterjee K, Daley J, Douglas JS, et al. (1999). ACC/AHA/ACP-ASIM guidelines for the management of patients with chronic stable angina: executive summary and recommendations. A Report of the American College of Cardiology/American Heart Association Task Force on Practice Guidelines (Committee on Management of Patients with Chronic Stable Angina). Circulation 99: 2829-2848.

Glynn RJ, Chae CU, Guralnik JM, Taylor JO, et al. (2000). Pulse pressure and mortality in older people. Arch. Intern. Med. 160: 2765-2772.

Huber C, Batchelor JR, Fuchs D, Hausen A, et al. (1984). Immune response-associated production of neopterin. Release from macrophages primarily under control of interferon-gamma. J. Exp. Med. 160: 310-316.

Monroe VS, Parilak LD and Kerensky RA (2002). Angiographic patterns and the natural history of the vulnerable plaque. Prog. Cardiovasc. Dis. 44: 339-347.

Naghavi M, Libby P, Falk E, Casscells SW, et al. (2003). From vulnerable plaque to vulnerable patient: a call for new definitions and risk assessment strategies: Part I. Circulation 108: 1664-1672.

Schmitz G, Herr AS and Rothe G (1998). T-lymphocytes and monocytes in atherogenesis. Herz 23: 168-177.

Genetics and Molecular Research 12 (4): 4222-4229 (2013)

CFUNPEC-RP www.funpecrp.com.br 
Ueland T, Vissers MN, Wiegman A, Rodenburg J, et al. (2006). Increased inflammatory markers in children with familial hypercholesterolaemia. Eur. J. Clin. Invest. 36: 147-152.

Weissberg PL (2000). Atherogenesis: current understanding of the causes of atheroma. Heart 83: 247-252.

Werle M, Schmal U, Hanna K and Kreuzer J (2002). MCP-1 induces activation of MAP-kinases ERK, JNK and p38 MAPK in human endothelial cells. Cardiovasc. Res. 56: 284-292.

Wirleitner B, Reider D, Ebner S, Bock G, et al. (2002). Monocyte-derived dendritic cells release neopterin. J. Leukoc. Biol. 72: 1148-1153. 